University of Shizuoka

US-00-08

August 2000

hep-ph/0008129

\title{
Bimaximal Neutrino Mixing in a Zee-type Model with Badly Broken Flavor Symmetry
}

\author{
Yoshio Koidef and Ambar Ghosall \\ Department of Physics, University of Shizuoka \\ 52-1 Yada, Shizuoka 422-8526, Japan
}

\begin{abstract}
A Zee-type neutrino mass matrix model with a badly broken horizontal symmetry $\mathrm{SU}(3)_{H}$ is investigated. By putting a simple ansatz on the symmetry breaking effects of $\mathrm{SU}(3)_{H}$ for transition matrix elements, it is demonstrated that the model can give a nearly bimaximal neutrino mixing with the ratio $\Delta m_{\text {solar }}^{2} / \Delta m_{\text {atm }}^{2} \simeq \sqrt{2} m_{e} / m_{\mu}=6.7 \times 10^{-3}$, which are in excellent agreement with the observed data. In the near future, the lepton-number violating decay $Z \rightarrow \mu^{ \pm} \tau^{\mp}$ will be observed.
\end{abstract}

PACS numbers: 14.60.Pq, 14.60.St, 11.30.Hv

* E-mail: koide@u-shizuoka-ken.ac.jp

$\dagger$ E-mail: gp1195@mail.a.u-shizuoka-ken.ac.jp 


\section{Introduction}

The recent Super-Kamiokande collaboration [1] has reported, by comparing the day/night spectrum and results of flux global analysis, that the small mixing angle MSW and justso solutions for active neutrinos are disfavored at 95\% C.L. and a mixing with sterile neutrinos is also disfavored at 95\% C.L. On the other hands, we have already known that the atmospheric neutrino data suggests a $\nu_{\mu} \leftrightarrow \nu_{\tau}$ mixing with a large mixing angle $\sin ^{2} 2 \theta \simeq 1$ [2]. If we take these experimental results seriously, we are forced to accept only a model which gives a nearly bimaximal mixing among the active neutrinos $\left(\nu_{e}, \nu_{\mu}, \nu_{\tau}\right)$. We must seek for the origin of the nearly bimaximal mixing.

As promising one of such the models, the Zee model [3] is known. In this model, a charged scalar field $h^{+}$is introduced in addition to the Higgs doublets $\Phi_{1}$ and $\Phi_{2}$;

$$
\begin{gathered}
\mathcal{L}=\frac{1}{2} \sum_{i, j} f_{i j} \bar{\ell}_{i L} i \tau_{2} \ell_{i L}^{c} h^{-}+c_{12} \Phi_{1}^{T} i \tau_{2} \Phi_{2} h^{-}+\sum_{i} y_{i} \bar{\ell}_{i L} \Phi_{2} e_{i R}+\text { h.c. } \\
\ell_{i L}=\left(\begin{array}{c}
\nu_{i L} \\
e_{i L}
\end{array}\right), \quad \Phi_{a}=\left(\begin{array}{c}
\Phi_{a}^{+} \\
\Phi_{a}^{0}
\end{array}\right), \quad i \tau_{2}=\left(\begin{array}{cc}
0 & 1 \\
-1 & 0
\end{array}\right),
\end{gathered}
$$

where $i=1,2,3$ are family indexes, $\ell_{i L}^{c}=\left(\ell_{i L}\right)^{c}=C \bar{\ell}_{i L}^{T}$, and $c_{12}=-c_{21}$ are real mass parameter. The neutrino mass matrix $M$ is radiatively generated as

$$
M_{i j}=m_{0} f_{i j}\left(m_{e j}^{2}-m_{e i}^{2}\right) / m_{\tau}^{2},
$$

where

$$
\begin{gathered}
m_{0}=\frac{\sin 2 \phi \tan \beta m_{\tau}^{2}}{32 \pi^{2} v / \sqrt{2}} \ln \frac{M_{1}^{2}}{M_{2}^{2}}, \\
\sin 2 \phi=-\frac{2 c_{12} v / \sqrt{2}}{M_{1}^{2}-M_{2}^{2}},
\end{gathered}
$$

$m_{e i}=y_{i}\left\langle\Phi_{2}^{0}\right\rangle=y_{i}(v / \sqrt{2}) \cos \beta, \tan \beta=\left\langle\Phi_{1}^{0}\right\rangle /\left\langle\Phi_{2}^{0}\right\rangle, v / \sqrt{2}=\sqrt{\left|\left\langle\Phi_{1}^{0}\right\rangle\right|^{2}+\left|\left\langle\Phi_{2}^{0}\right\rangle\right|^{2}}=174$ $\mathrm{GeV}$, and $M_{1}$ and $M_{2}$ are the masses of the charged scalars $H_{1}^{+}$and $H_{2}^{+}$, respectively, which are mass eigenstates of $\left(h^{+}, \Phi^{+}\right)$, i.e.,

$$
\begin{aligned}
\left(\begin{array}{c}
H_{1}^{+} \\
H_{2}^{+}
\end{array}\right) & =\left(\begin{array}{cc}
\cos \phi & -\sin \phi \\
\sin \phi & \cos \phi
\end{array}\right)\left(\begin{array}{c}
h^{+} \\
\Phi^{+}
\end{array}\right), \\
\Phi^{+} & =\Phi_{1}^{+} \cos \beta-\Phi_{2}^{+} \sin \beta
\end{aligned}
$$


Based on the recent solar and atmospheric neutrino data, Smirnov and Tanimoto [4] have investigated the model in detail, and they have concluded that there is no solution of the solar neutrino problem unless introducing a sterile neutrino $\nu_{s}$, although the model can explain the observed large mixing $\sin ^{2} 2 \theta_{\text {atm }} \simeq 1$. However, they have investigated only the case with $\varepsilon=M_{12} / \sqrt{M_{23}^{2}+M_{13}^{2}} \ll 1$ and $\tan \theta=M_{13} / M_{23} \ll 1$ considering $\Delta m_{21}^{2} \ll \Delta m_{32}^{2}\left(m_{1}<m_{2}<m_{3}\right)$, and they have not considered the case $\left|M_{12}\right| \sim\left|M_{13}\right| \gg$ $\left|M_{23}\right|$. The case $\left|M_{12}\right| \sim\left|M_{13}\right| \gg\left|M_{23}\right|$ has been investigated by Jarlskog et al. [5], and they have pointed out that the case can lead to the nearly bimaximal mixing.

In the present paper, we put a simple ansatz on the coupling constants $f_{i j}$ and $y_{i}$ under a badly broken flavor symmetry, and thereby we will obtain the nearly bimaximal mixing together with a prediction $\Delta m_{\text {solar }}^{2} / \Delta m_{\text {atm }}^{2} \simeq \sqrt{2} m_{e} / m_{\mu}=6.7 \times 10^{-3}$.

\section{Assumption on the symmetry breaking of $\mathrm{SU}(3)_{H}$}

We consider a badly broken horizontal symmetry [6] $\mathrm{SU}(3)_{H}$. We introduce parameters $s_{i}(i=1,2,3)$ as a measure of the symmetry breaking of $\mathrm{SU}(3)_{H}$. In the present paper, we do not touch the origin of the symmetry breaking.

Our basic assumption on the magnitudes of the symmetry breaking effects is as follows: The magnitude of the matrix element $\left\langle e_{i}(\mathbf{p})\left|y_{i j}\left(\bar{e}_{i} e_{j}\right)\right| e_{j}(\mathbf{p})\right\rangle$, which is a component of $3 \times 3^{*}=1+8$ of SU $(3)_{H}$, is proportional to $\delta_{i j} s_{i}^{2}$ in the limit of $|\mathbf{p}| \rightarrow \infty$, i.e.,

$$
y_{i}\left\langle e_{i}(\mathbf{p})\left|\left(\bar{e}_{i} e_{i}\right)\right| e_{i}(\mathbf{p})\right\rangle=s_{i}^{2} \times \text { const },
$$

while the magnitude of the matrix element $\left\langle\bar{\nu}_{i L}(\mathbf{p})\left|f_{i j}\left(\bar{\nu}_{i L}^{c} e_{j L}\right)\right| e_{j}^{-}(\mathbf{p})\right\rangle-(i \leftrightarrow j)$, which is a component of $3^{*}$ of $\mathrm{SU}(3)_{H}$, is proportional to $\Sigma_{k} \varepsilon_{i j k} s_{k}$ in the limit of $|\mathbf{p}| \rightarrow \infty$, i.e.,

$$
f_{i j}\left\langle\bar{\nu}_{i L}(\mathbf{p})\left|\left(\bar{\nu}_{i L}^{c} e_{j L}\right)\right| e_{j}^{-}(\mathbf{p})\right\rangle-(i \leftrightarrow j)=\sum_{k} \varepsilon_{i j} s_{k} \times \text { const } .
$$

Here, note that our requirements are applied in the limit of $|\mathbf{p}| \rightarrow \infty$, because the state $e_{i L}$ ( also even $\nu_{i L}$ ) is not eigenstate of the helicity $h$ in finite momentum frame unless the particle is massless.

These matrix elements, the left-hand sides of Eqs. (2.1) and (2.2), are evaluated as follows:

$$
\begin{gathered}
y_{i} \frac{1}{(2 \pi)^{3}} \frac{m_{e i}}{E_{e i}} \bar{u}_{e i}(\mathbf{p}) u_{e i}(\mathbf{p}), \\
f_{i j} \frac{1}{(2 \pi)^{3}} \sqrt{\frac{m_{e j}}{E_{e j}}} \sqrt{\frac{m_{\nu i}}{E_{\nu i}}} \bar{u}_{\nu i L}^{c}(\mathbf{p}) u_{e j L}(\mathbf{p})-(i \leftrightarrow j),
\end{gathered}
$$


respectively, where the spinor $u_{i}(\mathbf{p})$ is normalized as $\bar{u}_{i}(\mathbf{p}) u_{i}(\mathbf{p})=1$. Since in the limit of $|\mathbf{p}| \rightarrow \infty$, we obtain $\bar{u}_{e i}(\mathbf{p}) u_{e i}(\mathbf{p})=1$ and

$$
\lim _{|\mathbf{p}| \rightarrow \infty} \bar{u}_{\nu i L}^{c}(\mathbf{p}) u_{e j L}(\mathbf{p})=\frac{m_{e j}}{2 \sqrt{m_{\nu i} m_{e j}}}
$$

so that the assumptions (2.1) and (2.2) require

$$
y_{i} m_{e i}=s_{i}^{2} \times \text { const }
$$

and

$$
f_{i j}\left(m_{e j}+m_{e i}\right)=\sum_{k} \varepsilon_{i j k} s_{k} \times \text { const }
$$

respectively, where "const" includes $|\mathbf{p}|$.

Note that even if we apply our ansatz to the terms $\sum_{i} y_{i}\left(\bar{\nu}_{i L} e_{i R}\right) \Phi^{+}+$h.c. instead of the terms $\sum_{i} y_{i}\left(\bar{e}_{i L} e_{i R}\right) \Phi^{0}+h . c$. which leads to the requirement (2.1), we can obtain the same result with the result (2.6) because of

$$
y_{i} \frac{1}{(2 \pi)^{3}} \sqrt{\frac{m_{\nu i}}{E_{\nu i}}} \sqrt{\frac{m_{e i}}{E_{e i}}} \bar{u}_{\nu i}(\mathbf{p}) u_{e i}(\mathbf{p}),
$$

and

$$
\lim _{|\mathbf{p}| \rightarrow \infty} \bar{u}_{\nu i L}(\mathbf{p}) u_{e i R}(\mathbf{p})=\frac{m_{e i}}{2 \sqrt{m_{\nu i} m_{e i}}}
$$

Recalling $y_{i}=m_{e i} /\left\langle\Phi_{2}^{0}\right\rangle$, from the results (2.6) and (2.7), we obtain the symmetry breaking effect on the coupling constants $f_{i j}$

$$
f_{i j} \propto \frac{\varepsilon_{i j k} s_{k}}{m_{e j}+m_{e i}} \propto \frac{\varepsilon_{i j k} m_{e k}}{m_{e j}+m_{e i}},
$$

i.e.,

$$
f_{e \mu}=\varepsilon_{123} \frac{m_{\tau}}{m_{\mu}+m_{e}} f, \quad f_{e \tau}=\varepsilon_{132} \frac{m_{\mu}}{m_{\tau}+m_{e}} f, \quad f_{\mu \tau}=\varepsilon_{231} \frac{m_{e}}{m_{\tau}+m_{\mu}} f .
$$

\section{Mass spectrum and bimaximal mixing}

By using the results (2.11), we obtain the following mass matrix elements $M_{i j}$ :

$$
\begin{aligned}
& M_{e \mu}=\varepsilon_{123} m_{\tau}\left(m_{\mu}-m_{e}\right) f m_{0} / m_{\tau}^{2}, \\
& M_{e \tau}=\varepsilon_{132} m_{\mu}\left(m_{\tau}-m_{e}\right) f m_{0} / m_{\tau}^{2},
\end{aligned}
$$




$$
M_{\mu \tau}=\varepsilon_{231} m_{e}\left(m_{\tau}-m_{\mu}\right) f m_{0} / m_{\tau}^{2}
$$

Since $m_{\tau} \gg m_{\mu} \gg m_{e}$, we obtain

$$
M \simeq\left(\begin{array}{ccc}
0 & a & -a \\
a & 0 & b \\
-a & b & 0
\end{array}\right)
$$

where

$$
a=\frac{m_{\mu}}{m_{\tau}} f m_{0}, \quad b=\frac{m_{e}}{m_{\tau}} f m_{0} .
$$

The matrix form (3.2), except for the sign of $M_{13}$, is identical with the neutrino mass matrix which has recently been proposed by one of the authors (A.G.) [7] on the basis of discrete $Z_{3} \times Z_{4}$ symmetries, and it is know that the matrix form (3.2) can lead to the nearly bimaximal mixing. The mixing matrix $U$ and mass eigenvalues $m_{\nu i}$ are as follows:

$$
\begin{gathered}
U=\left(\begin{array}{ccc}
\cos \theta & -\sin \theta & 0 \\
-\frac{1}{\sqrt{2}} \sin \theta & -\frac{1}{\sqrt{2}} \cos \theta & \frac{1}{\sqrt{2}} \\
\frac{1}{\sqrt{2}} \sin \theta & \frac{1}{\sqrt{2}} \cos \theta & \frac{1}{\sqrt{2}}
\end{array}\right), \\
\tan \theta=\left(\frac{\sqrt{8 a^{2}+b^{2}}+b}{\sqrt{8 a^{2}+b^{2}}-b}\right)^{1 / 2}=\sqrt{\frac{-m_{\nu 1}}{m_{\nu 2}}} \\
m_{\nu 1}=-\frac{1}{2}\left(\sqrt{8 a^{2}+b^{2}}+b\right), \quad m_{\nu 2}=\frac{1}{2}\left(\sqrt{8 a^{2}+b^{2}}-b\right), \quad m_{\nu 3}=b .
\end{gathered}
$$

Since $a \gg b$ in the present model, we obtain

$$
\begin{gathered}
\Delta m_{12}^{2}=m_{\nu 1}^{2}-m_{\nu 2}^{2}=b \sqrt{8 a^{2}+b^{2}} \simeq 2 \sqrt{2} a b, \\
\Delta m_{23}^{2}=m_{\nu 2}^{2}-m_{\nu 3}^{2} \simeq 2 a^{2},
\end{gathered}
$$

so that we can predict

$$
\frac{\Delta m_{12}^{2}}{\Delta m_{23}^{2}} \simeq \sqrt{2} \frac{b}{a}=\sqrt{2} \frac{m_{e}}{m_{\mu}}=6.7 \times 10^{-3}
$$

together with the (nearly) bimaximal mixing

$$
U \simeq\left(\begin{array}{ccc}
\frac{1}{\sqrt{2}} & -\frac{1}{\sqrt{2}} & 0 \\
-\frac{1}{2} & -\frac{1}{2} & \frac{1}{\sqrt{2}} \\
\frac{1}{2} & \frac{1}{2} & \frac{1}{\sqrt{2}}
\end{array}\right)
$$


We regard $\Delta m_{12}^{2}$ and $\Delta m_{23}^{2}$ as $\Delta m_{\text {solar }}^{2}$ and $\Delta m_{\text {atm }}^{2}$, respectively. The predicted value (3.9) is in excellent agreement with the observed value [8, 2]

$$
\left(\frac{\Delta m_{\text {solar }}^{2}}{\Delta m_{\text {atm }}^{2}}\right)_{\text {exp }} \simeq \frac{2.2 \times 10^{-5} \mathrm{eV}^{2}}{3.2 \times 10^{-3} \mathrm{eV}^{2}} \simeq 6.9 \times 10^{-3}
$$

Note that the neutrino mass hierarchy in the present model is $\left|m_{\nu 1}\right| \simeq m_{\nu 2} \gg m_{\nu 3}$. Since $\Delta m_{23}^{2} \simeq m_{\nu 2}^{2}$, we can obtain the value of $m_{\nu 2}$ (and $\left.m_{\nu 1}\right)$ as follows

$$
\left|m_{\nu 1}\right| \simeq\left|m_{\nu 2}\right| \simeq \sqrt{\Delta m_{23}^{2}} \simeq \sqrt{\Delta m_{a t m}^{2}} \simeq 5.7 \times 10^{-2} \mathrm{eV}
$$

so that we also obtain

$$
m_{\nu 3} \simeq \frac{b}{\sqrt{2} a} m_{\nu 2}=\frac{m_{e}}{\sqrt{2} m_{\mu}} m_{\nu 2}=2.0 \times 10^{-4} \mathrm{eV} .
$$

It will be hard to detect such small masses of $m_{\nu i}$ directly. Furthermore, since $\left\langle m_{\nu}\right\rangle \equiv$ $\left|\sum_{i} m_{i} U_{e i}^{2}\right|=0$ due to $M_{11}=0$ in the present model, it is also impossible to detect the effective mass $\left\langle m_{\nu}\right\rangle$ in the neutrinoless double beta decay.

From

$$
\Delta m_{a t m}^{2}=\Delta m_{23}^{2} \simeq 2 a^{2} \simeq\left(\frac{m_{\mu}}{m_{\tau}} f m_{0}\right)^{2}
$$

we obtain the numerical result

$$
\begin{gathered}
f m_{0} \simeq 2.4 \times 10^{-3} \mathrm{eV}, \\
f \sin 2 \phi \tan \beta \ln \frac{M_{1}^{2}}{M_{2}^{2}} \simeq 1.2 \times 10^{-5} .
\end{gathered}
$$

\section{Constraints from the electroweak data}

The sensitive upper bound on $\left|f_{i j}\right|$ is , at present, given from the $\mu \rightarrow e \bar{\nu}_{e} \nu_{\mu}$ decay as derived by Smirnov and Tanimoto [⿴囗

$$
\left|f_{e \mu}\right|^{2}<2.8 \times 10^{-3} G_{F} \bar{M}^{2}
$$

where $\bar{M}$ is defined by $\left(1 / \bar{M}^{2}\right)=\left(1 / M_{1}^{2}\right) \cos ^{2} \phi+\left(1 / M_{2}^{2}\right) \sin ^{2} \phi$ and $G_{F} \bar{M}^{2}=\sqrt{2}[\bar{M} /(v / \sqrt{2})]^{2}$, $(v / \sqrt{2}=174 \mathrm{GeV})$. (Our definition of the coupling constants $f_{i j}$ are different from that in Ref. [⿴囗十 by a factor 2.) From the relation

$$
\sin ^{2} \phi=\frac{M_{\Phi}^{2}-M_{2}^{2}}{M_{1}^{2}-M_{2}^{2}}
$$


where $M_{\Phi}^{2}$ is the squared mass of the charged scalar $\Phi^{+}$defined by Eq. (1.7) [the $M_{11}^{2}$ component of the mass matrix $M^{2}$ for $\left.\left(\Phi^{+}, h^{+}\right)\right]$, we estimate

$$
\bar{M}^{2}=\frac{M_{1}^{2} M_{2}^{2}}{M_{2}^{2} \cos ^{2} \phi+M_{1}^{2} \sin ^{2} \phi}=\frac{M_{1}^{2} M_{2}^{2}}{M_{\Phi}^{2}} \sim M_{1}^{2}
$$

for $M_{\Phi}^{2} \sim M_{2}^{2} \sim 10^{4} \mathrm{GeV}^{2}$. Therefore, we can read the constraint (4.1) as

$$
\left|f_{e \mu}\right|<\frac{M_{1}}{v / \sqrt{2}} \times 3 \times 10^{-2}
$$

Since we expect visible effects of the Zee boson, we consider a value of $f_{e \mu}$ as large as possible. For example, we suppose $f_{e \mu} \sim 10^{0}$, which means $f \sim 10^{-1}$ and $M_{1} \sim 10^{4} \mathrm{GeV}$. We consider that it is likely that the Zee boson has such a intermediate mass scale.

The contribution of the Zee boson to the radiative decays $\mu \rightarrow e \gamma, \tau \rightarrow e \gamma$ and $\tau \rightarrow \mu \gamma$ are proportional to $\left(f_{\mu \tau} f_{e \tau}\right)^{2},\left(f_{\tau \mu} f_{\mu e}\right)^{2}$ and $\left(f_{\tau e} f_{e \mu}\right)^{2}$, respectively, where

$$
\begin{gathered}
\left(f_{\mu \tau} f_{e \tau}\right)^{2} \simeq\left(\frac{m_{e}}{m_{\mu}}\right)^{2} f^{4} \simeq 2.3 \times 10^{-5} f^{4} \\
\left(f_{\tau \mu} f_{\mu e}\right)^{2} \simeq\left(\frac{m_{e} m_{\mu}}{m_{\tau}^{2}}\right)^{2} f^{4} \simeq 2.9 \times 10^{-10} f^{4}, \\
\left(f_{\tau e} f_{e \mu}\right)^{2} \simeq f^{4} .
\end{gathered}
$$

Although the dominant mode of the radiative decay in the present model is $\tau \rightarrow \mu \gamma$, the constraint on the Zee boson contribution in the $\mu \rightarrow e \gamma$ decay is still severe compared with that in the $\tau \rightarrow \mu \gamma$, because the present experimental upper limits [9] of the partial decay widths $\Gamma(\tau \rightarrow \mu \gamma)$ and $\Gamma(\mu \rightarrow e \gamma)$ are $B(\tau \rightarrow \mu \gamma) / \tau(\tau)<3.0 \times 10^{-6} / 2.9 \times$ $10^{-13} \mathrm{~s}=1.0 \times 10^{7} \mathrm{~s}^{-1}$ and $B(\mu \rightarrow e \gamma) / \tau(\mu)<4.9 \times 10^{-11} / 2.2 \times 10^{-6} \mathrm{~s}=2.2 \times 10^{-5} \mathrm{~s}^{-1}$, respectively. However, even in the $\mu \rightarrow e \gamma$ decay, as discussed in Refs. [4] and [5], the present experimental upper limit of the decay rate $B(\mu \rightarrow e \gamma)$ cannot give a severe constraint on the magnitudes of $f_{i j}$.

We think that the most promising test of the present model is the observation of a lepton-number violating decay $Z \rightarrow \tau^{ \pm} \mu^{\mp}$, which is caused through $Z \rightarrow \nu_{e}+\bar{\nu}_{e}$ and a triangle loop with exchange of the Zee boson [and also through $Z \rightarrow H_{a}^{+}+H_{a}^{-}(a=1,2)$ ]. Similar lepton-number violating decays $Z \rightarrow e_{i} \bar{e}_{j}(i \neq j)$ can be caused by the exchange of scalar fermions in a minimal SUSY standard model with explicitly broken $R$-parity via $L$-violation [10]. In the present model, it is characteristic that only the dominant mode 
is $Z \rightarrow \tau^{ \pm} \mu^{\mp}$, which is proportional to $\left(f_{e \mu} f_{e \tau}\right)^{2} \simeq f^{4}$. We roughly estimate the ratio $R=B\left(Z \rightarrow \tau^{ \pm} \mu^{\mp}\right) / B\left(Z \rightarrow e^{+} e^{-}\right)$as

$$
R=\frac{\Gamma\left(Z \rightarrow \mu^{ \pm} \tau^{\mp}\right)}{\Gamma\left(Z \rightarrow e^{+} e^{-}\right)} \sim\left(\frac{f_{e \mu} f_{e \tau}}{16 \pi^{2}} \ln \frac{M_{1}^{2}}{m_{Z}^{2}}\right)^{2} \sim 10^{-6}
$$

where we have supposed $f_{e \mu} \sim 1$, i.e., $f \sim 10^{-1}$. (We must calculate all related diagrams in order to remove the logarithmic divergence. More details of the $Z \rightarrow e_{i} \bar{e}_{j}$ decays will be given elsewhere.) The present experimental upper limit 9 is $B(Z \rightarrow \tau \mu) / B(Z \rightarrow$ $e \bar{e})<1.2 \times 10^{-5} / 3.367 \times 10^{-2}=3.6 \times 10^{-4}$. We think that the value $R \sim 10^{-6}$ is within the reach of our near future experiment.

Another interesting observable quantity is the mixing matrix element $U_{e 3}$. The direct numerical calculation from the expression (3.1) [not the approximate expression (3.2)] gives the value of the mixing matrix element $U_{e 3}$

$$
U_{e 3}=-1.64 \times 10^{-5}
$$

However, the value (4.7) is too small to detect even in the near future, since the present experimental upper bound [11] is $U_{e 3}<(0.22-0.14)$.

\section{Conclusion and discussion}

In conclusion, we have investigated a neutrino mass matrix based on the Zee model with a badly broken horizontal symmetry. A simple ansatz for the symmetry breaking effects leads to $f_{e \mu} \simeq\left(m_{\tau} / m_{\mu}\right) f, f_{e \tau} \simeq\left(m_{\mu} / m_{\tau}\right) f$ and $f_{\mu \tau} \simeq\left(m_{e} / m_{\tau}\right) f$ for the Zee bosonlepton coupling constants $f_{i j}$. The Zee mass matrix with such coupling constants $f_{i j}$ gives the nearly bimaximal mixing (3.10) and the ratio of the squared mass differences $\Delta m_{\text {solar }}^{2} / \Delta m_{\text {atm }}^{2}=\sqrt{2} m_{e} / m_{\mu}=6.7 \times 10^{-3}$.

Since the coupling constant $c_{12}$ of the $\Phi_{1}^{T} i \tau_{2} \Phi_{2} h^{-}$term in the Lagrangian (1.1) has a dimension of mass, we think that the Lagrangian (1.1) is not a fundamental one, but an effective one. In the present model, the horizontal symmetry $\mathrm{SU}(3)_{H}$ is badly broken. We do not consider that the broken symmetry in the effective Lagrangian is brought by a spontaneous symmetry breaking. We consider that there is no horizontal symmetry in the fundamental Lagrangian from the beginning. Nevertheless, we have used the prescription of the "broken symmetry" only for the convenience of the phenomenological treatments.

Usually, the assumptions for the symmetry breaking are put on the coupling constants directly, while in the present paper, the requirements are put on the transition matrix elements including the coupling constants. The present prescription is somewhat 
unfamiliar and strange if quarks and leptons are fundamental entities. The present assumption may be understood by a composite model picture of quarks and leptons in future.

The present phenomenological success seems to suggest that the Zee model should be taken seriously. Then, our future tasks will be as follows: What is the meaning of the present prescription for the symmetry breaking? How can the Zee model we embedded into a unification scenario?

\section{Acknowledgments}

We would like to thank Professor M. Matsuda for his helpful discussions, especially, for offering us his detailed calculation on the $\mu \rightarrow e \gamma$ decay. One of the authors (Y.K.) thanks M. Tanimoto for his valuable comments and for informing useful references. One of the authors (A.G.) is supported by the Japan Society for Promotion of Science (JSPS), Postdoctoral Fellowship for Foreign Researches in Japan (Grant No. 99222).

\section{References}

[1] Y. Suzuki, Talk presented at Neutrino 2000, Sudbury, Canada, June 2000 (http://nu2000.sno.laurentian.ca/).

[2] Y. Fukuda et al., Phys. Lett. B335, 237 (1994); Super-Kamiokande collaboration, Y. Fukuda, et. al., Phys. Rev. Lett. 81, 1562 (1998); H. Sobel, Talk presented at Neutrino 2000, Sudbury, Canada, June 2000 (http://nu2000.sno.laurentian.ca).

[3] A. Zee, Phys. Lett. 93B, 389 (1980).

[4] A. Yu. Smirnov and M. Tanimoto, Phys. Rev. D55, 1665 (1997).

[5] C. Jarlskog, M. Matsuda, S. Skadhauge, M. Tanimoto, Phys. Lett. B449, 240 (1999).

[6] K. Akama and H. Terazawa, Univ. of Tokyo, Report No.257, 1976 (unpublished); T. Maehara and T. Yanagida, Prog. Theor. Phys. 60, 822 (1978); F. Wilczek and A. Zee, Phys. Rev. Lett. 42, 421 (1979) ; A. Davidson, M. Koca and K. C. Wali, Phys. Rev. D20, 1195 (1979); J. Chakrabarti, ibid. D20, 2411 (1979).

[7] A. Ghosal, to be published in Phys. Rev. D62, 0920xx (2000).

[8] M. Gonzalez-Garcia, Talk presented at Neutrino 2000, Sudbury, Canada, June 2000 (http://nu2000.sno.laurentian.ca/).

[9] Particle data group, Eur. Phys. Jour. C15, 1 (2000) (http://pdg.lbl.gov). 
[10] M. A. Mughal, M. Sadiq and K. Ahmed, Phys. Lett. B417, 87 (1998).

[11] CHOOZ Collaboration, M. Apollonio et al., Report No. hep-ex/9907037. 